\title{
A Family of Left Lie Bol Loops
}

\author{
Alper Bulut ${ }^{1}$ \\ ${ }^{1}$ Department of Mathematics, American University of the Middle East, College of Engineering and Technology, Kuwait \\ Correspondence: Alper Bulut, Department of Mathematics, American University of the Middle East, College of Engi- \\ neering and Technology, Kuwait. Email: alper.bulut@aum.edu.kw
}

Received: January 3, $2017 \quad$ Accepted: January 20, $2017 \quad$ Online Published: March 5, 2017
$\begin{array}{ll}\text { doi:10.5539/jmr.v9n2p1 } & \text { URL: https://doi.org/10.5539/jmr.v9n2p1 }\end{array}$

\section{Abstract}

In this paper the left Bol split extension method is used to build left Bol Lie loops from the Lie groups $H$ and $K$ such that $H$ is a Lie subgroup of $\operatorname{Aut}(K)$. Furthermore, we investigated some of the properties of those loops constructed in this way. Examples are given for finite and infinite dimensional left Bol Lie loops. Moreover, we showed that the twisted semidirect product of Lie algebras is an Akivis algebra.

Keywords: loops and quasigroups, semidirect product, twisted semidirect product, Lie loops.

\section{Introduction}

Non-associative semidirect product of groups are investigated in the last decades intensively in (Johnson \& Sharma,1980; Kinyon \& Jones, 2000; Nagy \& Strambach, 2008; Johnson \& Smith, 2010; Greer \& Raney, 2014). The twisted semidirect product of groups is also a known object in loop theory, its first appearance is in (Johnson \& Sharma, 1980), where they called the method the left Bol split extension. The construction method was further surveyed and generalized by Johnson and Smith (Johnson \& Smith, 2010). In this paper the left Bol Lie loops that are formed by the twisted semidirect product of Lie groups are investigated. We also explore examples of infinite dimensional left Bol Lie loops that are raised by the action of Lie subgroups of $G L(\mathcal{H})$ on the Hilbert space $\mathcal{H}$ over $\mathbb{C}$.

It is well-known that semidirect product of Lie algebras is a Lie algebra, so we naturally asked this question for the twisted semidirect product of Lie algebras of the Lie groups. We showed that twisted semidirect product of Lie algebras of the Lie groups is a Lie algebra which turned out to an Akivis algebra.

\section{Preliminaries}

We use the function evaluation in the backwards. If $\alpha: X \rightarrow Y$ is a function, then the function evaluation of $\alpha$ at the point $x \in X$ is denoted by $(x) \alpha$ or $x^{\alpha}$. Let $\beta: Y \rightarrow Z$ be another function, then the composition of $\alpha$ and $\beta$ is the function $\gamma:=\alpha \beta$ such that $(x)^{\alpha \beta}:=\left(x^{\alpha}\right)^{\beta}$ for all $x$ in the domain of $\alpha$. Let $G$ be a group. The elements $a, b$ of $G$ is said to conjugate if there exists a $g \in G$ such that $g^{-1} a g=b$ where $g^{-1} a g:=a^{g}$.

The nonempty set $L$ with a binary operation, $\oplus$, is called a loop if there exists $e \in L$ such that for all $a \in L a \oplus e=e \oplus a=a$, and the equations $a \oplus x=b$ and $y \oplus a=b$ have always unique solutions $x:=a \backslash b$ and $y:=b / a$ in $L$ whenever $a$ and $b$ are given in $L$. The uniqueness of $x$ and $y$ lead us to define two new maps that are called the left division $\backslash: L \times L \rightarrow L$ $(a, b) \mapsto a \backslash b$, and the right division $/: L \times L \rightarrow L(a, b) \mapsto b / a$ such that $a \oplus(a \backslash b)=b$ and $(b / a) \oplus a=b$.

Let $(L, \oplus)$ be a loop. Given any $x \in L$, let $L_{x}: L \rightarrow L$ and $R_{x}: L \rightarrow L$ be two maps defined by $(a) R_{x}:=a \oplus x,(b) L_{x}:=x \oplus b$ where $a, b \in L$. The maps $L_{x}$ and $R_{x}$ are called the left and the right translation maps respectively for $x$. It is well known that if $(L, \oplus)$ is a loop, then the left and the right translation maps are bijective. The loop $(L, \oplus)$ is called a left Bol loop if the left Bol identity given in (1) is valid for all $a, b$, and $c$ in $L$ :

$$
a \oplus(b \oplus(a \oplus c))=(a \oplus(b \oplus a)) \oplus c .
$$

Further readings on Bol loops can be found in (Robinson, 1966; Pflugfelder, 1990; Kiechle 2002). Next we define some groups acting on $L$, namely right multiplication group and left multiplication group. Right multiplication group, $\operatorname{Rmlt}(L)$, of $L$ is the permutation group generated by all right translations of $L$. The left multiplication group, $L m l t(L)$, is defined similarly. The multiplication group of $L, \operatorname{Mlt}(L)$, is the permutation group generated by all right and left translations of $L$. Hence, $\operatorname{Mlt}(L)=\left\langle L_{a}, R_{b}: a, b \in L\right\rangle$.

$G$ is called a Lie group if $G$ is a group and $G$ is a smooth manifold such that multiplication and inversion maps are smooth (Knapp, 2016). A Lie loop $L$ is a loop and a smooth manifold such that multiplication, right and left division maps are all smooth (Nagy \& Strambach, 2008). In this paper we mainly focus on the examples of Lie loops that are obtained from the twisted semidirect product of matrix Lie groups. 
Let $M(n, \mathbb{C})$ be the set of matrices of size $n$ by $n$ with complex entries and let $G L(n, \mathbb{C})$ be the general linear group. A matrix Lie group is a closed subgroup of $G L(n, \mathbb{C})$. The list of matrix Lie groups can be found in (Wallach, 1988; Hein, 1990). The left, the right and the middle nuclei of $(L, \oplus)$ can be defined respectively as follows:

$$
\begin{aligned}
N_{l} & =\{a \in L \mid(a \oplus x) \oplus y=a \oplus(x \oplus y) ; \forall x, y \in L\} . \\
N_{r} & =\{a \in L \mid(x \oplus y) \oplus a=x \oplus(y \oplus a) ; \forall x, y \in L\} . \\
N_{m} & =\{a \in L \mid(x \oplus a) \oplus y=x \oplus(a \oplus y) ; \forall x, y \in L\} .
\end{aligned}
$$

Note that $N_{l}, N_{r}$, and $N_{m}$ are all subgroups of $L$. The nucleus, $N(L)$, and the centrum, $C(L)$, of $(L, \oplus)$ are defined as follow:

$$
\begin{aligned}
& N(L):=N_{l} \cap N_{r} \cap N_{m} . \\
& C(L):=\{x \in L \mid x \oplus y=y \oplus x \forall y \in L\} .
\end{aligned}
$$

The center of $L$ is denoted by $Z(L)$ such that $Z(L):=C(L) \cap N(L)$. It is well known that the nucleus and the center of $L$ are subgroups of $L$, see (Pflugfelder, 1990).

Let $G$ be a group and $A$ be a set, and suppose $G$ acts on $A$ from the right. We use $A f f(A, G)$ to denote the set of maps, $f_{(a, g)}$, such that $(b) f_{(a, g)}=a+b . g$ where $a, b \in A$ and $g \in G$. If $G$ acts on $A$ by function evaluation, then $(b) f_{(a, g)}=a+b^{g}$.

\subsection{Semidirect and Twisted Semidirect Products}

Let $H$ and $K$ be groups such that $H \leq A u t(K)$ and consider $G:=K \times H$ as a set and define the multiplication, $\odot$, on $G$ as follow:

$$
\left(k_{1}, h_{1}\right) \odot\left(k_{2}, h_{2}\right)=\left(k_{1} k_{2}{ }^{h^{-1}}, h_{1} h_{2}\right) .
$$

Note that we used juxtapositions for the product in $K$ and $H$. It is well known that $(G, \odot)$ is a new group with the identity element $\left(e_{K}, e_{H}\right)$. The product given in (7) is called semidirect product of $K$ by $H$ and denoted by $G=K \rtimes H$, see (Hall, 1999). Note that if $H$ is acting on $K$ trivially, then the semidirect product is the usual direct product. The sets $K_{G}=\left\{\left(k, e_{H}\right): k \in K\right\}$ and $H_{G}=\left\{\left(e_{K}, h\right): h \in H\right\}$ have a trivial intersection, and $K_{G} \cong K$ and $H_{G} \cong H$ such that $K_{G}$ is normal subgroup of $G$.

If we replace $h_{1}^{-1}$ with $h_{1}$ in (7), then we obtain a new binary operation $\bar{\odot}$ on $K \times H$ as given in (8). Johnson and Sharma (1980) named the $K \times H$ with $\odot$ the left Bol split extension and they showed that if $H$ is a non-abelian group of $\operatorname{Aut}(K)$, then $(K \times H, \bar{\odot})$ is a left Bol loop. In the current paper we use the term twisted semidirect product for the binary operation $\bar{\odot}$.

$$
\left(k_{1}, h_{1}\right) \bar{\odot}\left(k_{2}, h_{2}\right)=\left(k_{1} k_{2}{ }^{h_{1}}, h_{1} h_{2}\right) .
$$

Each element of $H$ is an automorphism from $K$ to $K$ and the notation $k_{2}{ }^{h_{1}}$ stands for the image of $k_{2}$ under $h_{1}$. In general the twisted semidirect product is not necessarily associative.

\subsection{Lie Algebra and Akivis Algebra}

A Lie algebra, see (Humphreys, 1972; Knapp, 2016), is a vector space $\mathfrak{g}$ over a field $\mathbb{F}$ that endowed with bracket operation $[.,]:. \mathfrak{g} \times \mathfrak{g} \rightarrow \mathfrak{g},(x, y) \mapsto[x, y]$ that satisfies the following axioms:

1. The bracket operation is bilinear.

2. $[x, x]=0$ for all $x \in \mathfrak{g}$.

3. $[[x, y], z]+[[y, z], x]+[[z, x], y]=0$.

The last axiom is called the Jacobi identity. Note that combining the first two axioms yields that $[x, y]=-[y, x]$ for all $x, y \in \mathfrak{g}$. Therefore, the bracket operation is skew-symmetric in a Lie algebra. A homomorphism, $\phi$, of Lie algebras from $\mathfrak{g}_{1}$ to $\mathfrak{g}_{2}$ is a linear map that preserves the brackets that is $([x, y]) \phi=[(x) \phi,(y) \phi]$ for $x, y \in \mathfrak{g}_{1}$. A derivation of a Lie algebra $\mathfrak{g}$ over a field $\mathbb{F}$ is a linear map $f: \mathfrak{g} \rightarrow \mathfrak{g}$ such that $([x, y]) f=[(x) f, y]+[x,(y) f]$ for all $x, y \in \mathfrak{g}$ (Hein, 1990). The set of all derivation of $\mathfrak{g}$ over $\mathbb{F}$ is denoted by $\operatorname{Der}_{\mathbb{F}}(\mathfrak{g})$. The derivation is a Lie algebra if the bracket operation is defined as $[f, g]=g f-f g$ for $f, g \in \operatorname{Der}_{\mathbb{F}}(\mathfrak{g})$. A Lie subalgebra $\mathfrak{h}$ is a vector subspace of $\mathfrak{g}$ such that $\mathfrak{h}$ is closed under bracket operation. A Lie subalgebra $\mathfrak{h}$ of $\mathfrak{g}$ is called an ideal of $\mathfrak{g}$ if $[\mathfrak{h}, \mathfrak{g}] \subseteq \mathfrak{h}$.

An Akivis algebra $(\mathcal{A},[.,],.\langle., .,\rangle$.$) is a real vector space with a bilinear skew-symmetric map (x, y) \mapsto[x, y]: \mathcal{A} \times \mathcal{A} \rightarrow \mathcal{A}$, called the commutator map, and a trilinear map $(x, y, z) \mapsto\langle x, y, z\rangle: \mathcal{A} \times \mathcal{A} \times \mathcal{A} \rightarrow \mathcal{A}$, called the associator map, such that the following identity (called the Akivis identity) holds (Figula \& Strambach, 2007). 


$$
[[x, y], z]+[[y, z], x]+[[z, x], y]=\langle x, y, z\rangle+\langle y, z, x\rangle+\langle z, x, y\rangle-(\langle x, z, y\rangle+\langle y, x, z\rangle+\langle z, y, x\rangle)
$$

If $\mathcal{A}$ is a Lie algebra, then the left hand-side of the equality is zero by the Jacobi identity.

Let $\mathfrak{k}, \mathfrak{h}$ be Lie algebras over the same field $\mathbb{F}$, and suppose $\rho: \mathfrak{h} \rightarrow \operatorname{Der}_{\mathbb{F}}(\mathfrak{k}), h \mapsto(h) \rho$ and $k \mapsto(k)^{(h) \rho}$, be a Lie algebra homomorphism. The set $\mathfrak{k} \times \mathfrak{h}$ is a Lie algebra endowed with the bracket operation given in (10).

$$
\left[\left(k_{1}, h_{1}\right),\left(k_{2}, h_{2}\right)\right]=\left(\left[k_{1}, k_{2}\right]+\left(k_{1}\right)^{\left(h_{2}\right) \rho}-\left(k_{2}\right)^{\left(h_{1}\right) \rho},\left[h_{1}, h_{2}\right]\right)
$$

The new Lie algebra with the bracket defined in (10) is called a semidirect product of $\mathfrak{k}$ and $\mathfrak{h}$, and it is denoted by $\mathfrak{k} \rtimes_{\rho} \mathfrak{l}$. The following result is well known; see for example (Hein, 1990).

Theorem 2.1. Let $\mathfrak{h}$ and $\mathfrak{k}$ be two Lie algebras over the field $\mathbb{F}$, and let $\rho: \mathfrak{h} \rightarrow \operatorname{Der}_{\mathbb{F}}(\mathfrak{k})$ be a Lie algebra homomorphism. Then,

1. $\mathfrak{l}=\mathfrak{k} \rtimes_{\rho} \mathfrak{h}$ is a Lie algebra

2. $\overline{\mathfrak{k}}=\{(k, 0): k \in \mathfrak{k}\} \cong \mathfrak{k}$ such that $\mathfrak{k}$ is an ideal of $\mathfrak{l}$, i.e., $[\overline{\mathfrak{k}}, \mathfrak{l}] \subseteq \overline{\mathfrak{k}}$.

3. $\overline{\mathfrak{h}}=\{(0, h): h \in \mathfrak{h}\} \cong \mathfrak{h}$ such that $\mathfrak{h}$ is a subalgebra of $\mathfrak{l}$, i.e., $[\overline{\mathfrak{h}}, \overline{\mathfrak{h}}] \subseteq \overline{\mathfrak{h}}$.

We obtain a new bracket operation from (10) by interchanging $k_{1}{ }^{h_{2}}$ and $k_{2}{ }^{h_{1}}$ in (10). We call this new bracket operation, given in (11), the twisted semidirect product of $\mathfrak{k}$ and $\mathfrak{h}$. The set $\mathfrak{k} \times \mathfrak{h}$ with twisted semidirect product is denoted by $\mathfrak{k} \bar{\searrow}_{\rho} \mathfrak{h}$.

$$
\left[\left(k_{1}, h_{1}\right),\left(k_{2}, h_{2}\right)\right]=\left(\left[k_{1}, k_{2}\right]+\left(k_{2}\right)^{\left(h_{1}\right) \rho}-\left(k_{1}\right)^{\left(h_{2}\right) \rho},\left[h_{1}, h_{2}\right]\right)
$$

\section{Main Results}

Theorem 3.1. Let $H$ and $K$ be Lie groups with $H \leq A u t(K)$ such that the evaluation map ev : $K \times H \rightarrow K,(k, h) \mapsto k^{h}$ is smooth. If $\mathcal{L}:=(K \times H, \bar{\odot})$, then

1. $\mathcal{L}$ is a Lie group if and only if $H$ is an abelian Lie group.

2. If $H$ is not abelian, then $\mathcal{L}$ is a left Bol Lie loop, not a Lie group.

Proof. We first prove the first argument. If $\mathcal{L}$ is a Lie group, then its group product $\bar{\odot}$ is associative. That is the equation (12) holds for all $\left(k_{1}, h_{1}\right),\left(k_{2}, h_{2}\right)$, and $\left(k_{3}, h_{3}\right)$ in $\mathcal{L}$

$$
\left[\left(k_{1}, h_{1}\right) \bar{\odot}\left(k_{2}, h_{2}\right)\right] \bar{\odot}\left(k_{3}, h_{3}\right)=\left(k_{1}, h_{1}\right) \bar{\odot}\left[\left(k_{2}, h_{2}\right) \bar{\odot}\left(k_{3}, h_{3}\right)\right]
$$

The left hand side of the equation (12) is equal to $\left(k_{1} k_{2}^{h_{1}} k_{3}^{h_{1} h_{2}},\left(h_{1} h_{2}\right) h_{3}\right)$ and the right hand side is equal to $\left(k_{1} k_{2}^{h_{1}} k_{3} h_{2} h_{1}, h_{1}\left(h_{2} h_{3}\right)\right)$. Two sides are equal if and only if $k_{3}{ }^{h_{1} h_{2}}=k_{3}{ }^{h_{2} h_{1}}$, but this forces that $H$ is an abelian Lie group. Conversely, suppose that $H$ is an abelian Lie group, then $\mathcal{L}$ is a smooth manifold as a cartesian product of smooth manifolds $K \times H$. Let $e_{K}$ and $e_{H}$ be the identity elements of $K$ and $H$ respectively, then it can be verified that $\left(e_{K}, e_{H}\right)$ is the identity element of $\mathcal{L}$. Moreover, for any arbitrary element $(k, h)$ of $\mathcal{L}$ the following equation is satisfied, hence the two sided inverse of $(k, h)$ exists.

$$
(k, h) \bar{\odot}\left(\left(k^{-1}\right)^{h^{-1}}, h^{-1}\right)=\left(\left(k^{-1}\right)^{h^{-1}}, h^{-1}\right) \bar{\odot}(k, h)=\left(e_{K}, e_{H}\right) .
$$

The group product $\odot$ of $\mathcal{L}$ is associative as shown below:

$$
\begin{aligned}
{\left[\left(k_{1}, h_{1}\right) \bar{\odot}\left(k_{2}, h_{2}\right)\right] \bar{\odot}\left(k_{3}, h_{3}\right) } & =\left(k_{1} k_{2}^{h_{1}}, h_{1} h_{2}\right) \bar{\odot}\left(k_{3}, h_{3}\right) \\
& =\left(\left(k_{1} k_{2}^{h_{1}}\right) k_{3} h_{1} h_{2},\left(h_{1} h_{2}\right) h_{3}\right) \\
& =\left(\left(k_{1} k_{2}^{h_{1}}\right) k_{3} h_{2} h_{1},\left(h_{1} h_{2}\right) h_{3}\right) \\
& =\left(k_{1}\left(k_{2}^{h_{1}} k_{3}^{h_{2} h_{1}}\right), h_{1}\left(h_{2} h_{3}\right)\right) \\
& =\left(k_{1}\left(k_{2}^{h_{1}}\left(k_{3}{ }^{h_{2}}\right)^{h_{1}}\right), h_{1}\left(h_{2} h_{3}\right)\right) \\
& =\left(k_{1}\left(k_{2} k_{3}^{h_{2}}\right)^{h_{1}}, h_{1}\left(h_{2} h_{3}\right)\right) \\
& =\left(k_{1}, h_{1}\right) \bar{\odot}\left(k_{2} k_{3}^{h_{2}}, h_{2} h_{3}\right) \\
& =\left(k_{1}, h_{1}\right) \bar{\odot}\left[\left(k_{2}, h_{2}\right) \bar{\odot}\left(k_{3}, h_{3}\right)\right] .
\end{aligned}
$$


Note that we used the assumption that $H$ is abelian in (16) to write $h_{1} h_{2}=h_{2} h_{1}$, and we used the fact that $h_{1}$ is an automorphism over $K$ to write $k_{2}^{h_{1}} k_{3}^{h_{2} h_{1}}=\left(k_{2} k_{3}^{h_{2}}\right)^{h_{1}}$ in (19). We conclude that $\mathcal{L}$ satisfies all group axioms, so it is a group besides its smooth manifold structure. To show it is actually a Lie group requires to show that the group product and the inversion maps are smooth.

Let $\mu_{\mathcal{L}}: \mathcal{L} \times \mathcal{L} \rightarrow \mathcal{L}$ such that $\left(\left(k_{1}, h_{1}\right),\left(k_{2}, h_{2}\right)\right) \mu_{\mathcal{L}}=\left(k_{1} k_{2}{ }^{h_{1}}, h_{1} h_{2}\right)$ and $i: \mathcal{L} \rightarrow \mathcal{L}$ such that $(k, h) i=\left(\left(k^{-1}\right)^{h^{-1}}, h^{-1}\right)$. Let $\mu_{K}$ and $\mu_{H}$ be the multiplication maps of $K$ and $H$ respectively and let $i_{K}$ and $i_{H}$ be the inversion maps of $K$ and $H$. By assumption $\mu_{K}, \mu_{H}$ and $i_{K}, i_{H}$ are all smooth maps. Observe that

$$
\begin{aligned}
\left(\left(k_{1}, h_{1}\right),\left(k_{2}, h_{2}\right)\right) \mu_{\mathcal{L}} & =\left(\left(k_{1}, k_{2}^{h_{1}}\right) \mu_{K},\left(h_{1}, h_{2}\right) \mu_{H}\right) \\
& =\left(\left(\left(k_{1}\right) i d,\left(k_{2}, h_{1}\right) e v\right) \mu_{K},\left(h_{1}, h_{2}\right) \mu_{H}\right) \\
& =\left(\left(\left(k_{1},\left(k_{2}, h_{1}\right)(i d \times e v)\right) \mu_{K},\left(h_{1}, h_{2}\right) \mu_{H}\right)\right. \\
& =\left(\left(k_{1},\left(k_{2}, h_{1}\right)\right)\left((i d \times e v) \circ \mu_{K}\right),\left(h_{1}, h_{2}\right) \mu_{H}\right) \\
& =\left(\left(k_{1},\left(k_{2}, h_{1}\right)\right),\left(h_{1}, h_{2}\right)\right)\left((i d \times e v) \circ \mu_{K}\right) \times \mu_{H} .
\end{aligned}
$$

$\mu_{\mathcal{L}}$ is smooth since the direct product of smooth maps and composition of smooth maps are smooth. Similar to multiplication the inversion map is also smooth that can be shown below.

$$
\begin{aligned}
(k, h) i_{\mathcal{L}} & =\left(\left(k^{-1}\right)^{h^{-1}}, h^{-1}\right) \\
& =\left(\left((k) i_{K},(h) i_{H}\right) e v,(h) i_{H}\right) \\
& \left.=\left((k, h)\left(i_{K} \times i_{H}\right) \circ e v\right),(h) i_{H}\right) \\
& =((k, h), h))\left(\left(i_{K} \times i_{H}\right) \circ e v\right) \times i_{H} .
\end{aligned}
$$

Therefore, $\mathcal{L}$ is a Lie group.

For the proof of the second argument let $H$ be a non-abelian subgroup of $\operatorname{Aut}(K)$, then $(K \times H, \bar{\odot})$ is a left Bol loop which has been shown in (Johnson \& Sharma, 1980). For the convenience of readers we prefer to provide the proof. Suppose that $H$ is non-abelian, then there exists $h_{1}, h_{2} \in H$ such that $h_{1} h_{2} \neq h_{2} h_{1}$. That means there exists a $k_{3} \in K$ such that $k_{3}{ }^{h_{1} h_{2}} \neq k_{3}{ }^{h_{2} h_{1}}$, then for nonzero $k_{1}, k_{2} \in K,\left(k_{1} k_{2}{ }^{h_{1}}\right) k_{3}{ }^{h_{1} h_{2}} \neq\left(k_{1} k_{2}{ }^{h_{1}}\right) k_{3}{ }^{h_{2} h_{1}}$ which is equivalent to:

$$
\left[\left(k_{1}, h_{1}\right) \bar{\odot}\left(k_{2}, h_{2}\right)\right] \bar{\odot}\left(k_{3}, h_{3}\right) \neq\left(k_{1}, h_{1}\right) \bar{\odot}\left[\left(k_{2}, h_{2}\right) \bar{\odot}\left(k_{3}, h_{3}\right)\right] .
$$

Therefore, if $H$ is not abelian, then the product on $\mathcal{L}$ is not associative, so $\mathcal{L}$ is not a Lie group. On the other hand, $\mathcal{L}$ is a smooth manifold as the cartesian product of smooth manifolds $K$ and $H$. Moreover, for all $(k, h) \in \mathcal{L}$

$$
(k, h) \bar{\odot}\left(e_{K}, e_{H}\right)=\left(e_{K}, e_{H}\right) \odot(k, h)=(k, h)
$$

hence $\left(e_{K}, e_{H}\right)$ is the neutral element of $\mathcal{L}$. We can always find unique $\left(x_{k}, x_{h}\right)$ and $\left(y_{k}, y_{h}\right)$ in $\mathcal{L}$ that satisfy the given equations in (33) and (34).

$$
\begin{array}{lll}
\left(k_{1}, h_{1}\right) \bar{\odot}\left(x_{k}, x_{h}\right)=\left(k_{2}, h_{2}\right) & \text { where } \quad\left(x_{k}, x_{h}\right):=\left(k_{1}, h_{1}\right) \backslash\left(k_{2}, h_{2}\right) . \\
\left(y_{k}, y_{h}\right) \bar{\odot}\left(k_{2}, h_{2}\right)=\left(k_{1}, h_{1}\right) & \text { where } \quad\left(y_{k}, y_{h}\right):=\left(k_{1}, h_{1}\right) /\left(k_{2}, h_{2}\right) .
\end{array}
$$

The solutions $\left(x_{k}, x_{h}\right)=\left(\left(k_{1}^{-1} k_{2}\right)^{h_{1}^{-1}}, h_{1}^{-1} h_{2}\right)$ and $\left(y_{k}, y_{h}\right)=\left(\left(k_{1}\left(k_{2}^{-1}\right)^{h_{1} h_{2}^{-1}}, h_{1} h_{2}^{-1}\right)\right.$ can be derived easily. We conclude that $\mathcal{L}$ is a loop. To show it is a Lie loop we also need to show that the twisted semidirect product, the left and the right division maps are all smooth. Based on (33) and (34) the right and the left division maps are derived as follow:

$$
\begin{aligned}
& \text { \: } \mathcal{L} \times \mathcal{L} \rightarrow \mathcal{L} \quad \text { such that } \quad\left(\left(k_{1}, h_{1}\right),\left(k_{2}, h_{2}\right)\right) \mapsto\left(\left(k_{1}^{-1} k_{2}\right)^{h_{1}^{-1}}, h_{1}^{-1} h_{2}\right) \\
& \text { / : } \quad \mathcal{L} \times \mathcal{L} \rightarrow \mathcal{L} \quad \text { such that } \quad\left(\left(k_{1}, h_{1}\right),\left(k_{2}, h_{2}\right)\right) \mapsto\left(\left(k_{1}\left(k_{2}^{-1}\right)^{h_{1} h_{2}^{-1}}, h_{1} h_{2}^{-1}\right)\right.
\end{aligned}
$$

We have already showed in the proof of first argument that the twisted semidirect product is smooth. On the other hand, the left and the right division maps can be written as direct products of smooth maps, thus they are also smooth as given below:

$$
\begin{aligned}
& \left(k_{1}, h_{1}\right) \backslash\left(k_{2}, h_{2}\right)=\left(\left(\left(k_{1}, k_{2}\right), h_{1}\right),\left(h_{1}, h_{2}\right)\right)\left(\left(\left(i_{K} \times i d_{K}\right) \mu_{K}\right) \times i d_{H}\right) e v \times\left(i_{H} \times i d_{H}\right) \mu_{H} \\
& \left(k_{1}, h_{1}\right) /\left(k_{2}, h_{2}\right)=\left(k_{1},\left(k_{2},\left(h_{1}, h_{2}\right)\right)\right)\left(i d_{K} \times\left(\left(i_{K} \times\left(i d_{H} \times i_{H}\right) \mu_{H}\right) e v\right) \mu_{K} \times\left(i d_{H} \times i_{H}\right) \mu_{H}\right.
\end{aligned}
$$

We conclude that if $H$ is non-abelian, then $\mathcal{L}$ is a left Bol Lie loop that is not a Lie group. 
Notice that if we set $h_{1}=h_{2}$ in (35) and $k_{1}=k_{2}$ in (36), then the following corollary is obtained.

Corollary 3.1.1. Let $H$ and $K$ be groups such that $H \leq$ Aut $(K)$ and let $\mathcal{L}:=(K \times H, \bar{\odot})$. Then

1. $\left(k_{1}, h_{1}\right) \backslash\left(k_{2}, h_{2}\right)=\left(e_{K}, h_{1}^{-1} h_{2}\right)$ if $k_{1}=k_{2}$.

2. $\left(k_{1}, h_{1}\right) /\left(k_{2}, h_{2}\right)=\left(k_{1} k_{2}^{-1}, e_{H}\right)$ if $h_{1}=h_{2}$.

Example 3.2. Let $\mathbb{C}$ be the field of complex numbers and let $n$ be a positive integer. It is well known that $\mathbb{C}^{n}$ is an additive Lie group and $G L(n, \mathbb{C}) \cong$ Aut $\left(\mathbb{C}^{n}\right)$ after fixing a basis of $\mathbb{C}^{n}$. Suppose $\phi: G L(n, \mathbb{C}) \rightarrow$ Aut $\left(\mathbb{C}^{n}\right)$ is the isomorphism and $i: G \rightarrow G L(n, \mathbb{C})$ be the inclusion map, where $G$ is a non-abelian closed subgroup of $G L(n, \mathbb{C})$. A closed subgroup of $G L(n, \mathbb{C})$ is a Lie group, hence $G$ is a Lie group. The map $\Phi:=i \circ \phi$ is a homomorphism of Lie groups from $G$ to Aut $\left(\mathbb{C}^{n}\right)$. On the other hand, the evaluation map is smooth since matrix multiplication is smooth. Therefore, $\left(\mathbb{C}^{n} \times G, \bar{\odot}\right)$ is a left Bol Lie loop by Theorem 3.1.

Example 3.3. For any matrix $A \in M(n, \mathbb{C})$ we use $A^{\top}$ and $A^{*}$ to denote the transpose of $A$ and conjugate transpose of $A$ respectively. Let $p, q \in \mathbb{N}$ and $p+q \geq 1$ and let $\alpha:=\left[\begin{array}{cc}I_{p} & 0 \\ 0 & -I_{q}\end{array}\right]$ and $\beta:=\left[\begin{array}{cc}0 & I_{n} \\ -I_{n} & 0\end{array}\right]$, where $I_{p}, I_{q}$, and $I_{n}$ are the identity matrices. The pseudo-unitary group $U(p, q)$ and the symplectic group $S p(n, \mathbb{C})$ are well-known non-abelian classical Lie groups given below:

$$
\begin{aligned}
U(p, q) & =\left\{A \in G L(p+q, \mathbb{C}): A \alpha A^{\top}=\alpha\right\} . \\
S p(n, \mathbb{C}) & =\left\{A \in G L(2 n, \mathbb{C}): A \beta A^{*}=\beta\right\} .
\end{aligned}
$$

Similar to example 3.2, $\left(\mathbb{C}^{p+q} \times U(p, q), \bar{\odot}\right)$ and $\left(\mathbb{C}^{2 n} \times S p(n, \mathbb{C}), \bar{\odot}\right)$ are both left Bol Lie loops.

Example 3.4. Let $a, b$, and c be arbitrary real numbers. The Heisenberg group, $H$, consists of 3 by 3 matrices in form of

$$
\left[\begin{array}{lll}
1 & a & b \\
0 & 1 & b \\
0 & 0 & 1
\end{array}\right]
$$

Heisengerg group is a closed subgroup of $G L(3, \mathbb{R})$, so it is a matrix Lie group. The evaluation map, ev : $\mathbb{R}^{3} \times H \rightarrow \mathbb{R}^{3}$, $(v, A) \mapsto v^{\top} A$ is smooth. It can be checked that the matrix multiplication in $H$ is not commutative. Therefore, $\left(\mathbb{R}^{3} \times H, \odot\right)$ is a left Bol Lie loop.

Corollary 3.4.1. Let $V$ be either finite or infinite dimensional linear space over a field $\mathbb{F}$ and let $G$ be any non-abelian subgroup of Aut $(V)$, then $\mathcal{L}:=(V \times G, \bar{\odot})$ is a left Bol loop such that:

1. $\operatorname{Lmlt}(\mathcal{L}) \subseteq \operatorname{Aff}(V, G) \times G$.

2. $N(\mathcal{L})=\{0\} \times Z(G)$.

3. $Z(\mathcal{L})=\{(0, i d)\}$.

Proof. $\mathcal{L}$ is a left Bol loop is immediate by Theorem 3.1 since $G$ is a non-abelian subgroup of $A u t(V)$ and $V$ is a linear space that means $V$ is an additive group. The twisted semidirect product over $V \times G$ is written as below:

$$
\left(v_{1}, g_{1}\right) \bar{\odot}\left(v_{2}, g_{2}\right)=\left(v_{1}+\left(v_{2}{ }^{g_{1}}\right), g_{1} g_{2}\right)
$$

We first prove (1). Let $L_{(v, g)}$ be any left translation of $\operatorname{Lmlt}(\mathcal{L})$, and let $(w, h)$ be any element of $\mathcal{L}$. Then

$$
\begin{aligned}
(w, h) L_{(v, g)} & =(v, g) \bar{\odot}(w, h) \\
& =\left(v+w^{g}, g h\right) \\
& =\left((w) \phi_{(v, g)},(h) L_{g}\right) \\
& =(w, h)\left(\phi_{(v, g)} \times L_{g}\right) .
\end{aligned}
$$

For each $(w, h) \in \mathcal{L},(w, h) L_{(v, g)}=(w, h)\left(\phi_{(v, g)} \times L_{g}\right)$, thus $L_{(v, g)}=\phi_{(v, g)} \times L_{g}$, and this implies $\operatorname{Lmlt}(\mathcal{L}) \subseteq A f f(V, G) \times \operatorname{Lmlt}(G)$. Note that $\operatorname{Lmlt}(G)=G$ since $G$ is a group, so $\operatorname{Lmlt}(\mathcal{L}) \subseteq A f f(V, G) \times G$. 
To see (2), we will determine the left, the middle and the right nuclei of $\mathcal{L}$. Let $\left(v_{1}, g_{1}\right)$ and $\left(v_{2}, g_{2}\right)$ be arbitrary elements of $\mathcal{L}$.

$$
\begin{aligned}
N_{l}(\mathcal{L}) & =\left\{(w, h) \in \mathcal{L}:\left[(w, h) \bar{\odot}\left(v_{1}, g_{1}\right)\right] \bar{\odot}\left(v_{2}, g_{2}\right)=(w, h) \bar{\odot}\left[\left(v_{1}, g_{1}\right) \bar{\odot}\left(v_{2}, g_{2}\right)\right]\right\} \\
& =\left\{(w, h) \in \mathcal{L}:\left(w+v_{1}{ }^{h}, h g_{1}\right) \bar{\odot}\left(v_{2}, g_{2}\right)=(w, h) \bar{\odot}\left(v_{1}+v_{2}{ }^{g_{1}}, g_{1} g_{2}\right)\right\} \\
& =\left\{(w, h) \in \mathcal{L}:\left(w+v_{1}{ }^{h}+v_{2}{ }^{h g_{1}},\left(h g_{1}\right) g_{2}\right)=\left(w+v_{1}{ }^{h}+v_{2}{ }^{g_{1} h}, h\left(g_{1} g_{2}\right)\right)\right\} \\
& =\left\{(w, h) \in \mathcal{L}: v_{2}{ }^{g_{1}}=v_{2}{ }^{g_{1} h}\right\} .
\end{aligned}
$$

The condition $v_{2}{ }^{h g_{1}}=v_{2}{ }^{g_{1} h}$ is independent from $w$, so $w$ can be anything in $V$. Moreover, if $v_{2}{ }^{h g_{1}}=v_{2}{ }^{g_{1} h}$ for all $v_{2}$ in $V$, then $h g_{1}=g_{1} h$ for all $g_{1} \in G$, thus $h \in Z(G)$. Therefore, $N_{l}(\mathcal{L})=V \times Z(G)$. In left Bol loops the left and the right nuclei are same (Robinson, 1966), hence we only need to find $N_{r}(\mathcal{L})$.

$$
\begin{aligned}
N_{r}(\mathcal{L}) & =\left\{(w, h) \in \mathcal{L}:\left[\left(v_{1}, g_{1}\right) \bar{\odot}\left(v_{2}, g_{2}\right)\right] \bar{\odot}(w, h)=\left(v_{1}, g_{1}\right) \bar{\odot}\left[\left(v_{2}, g_{2}\right) \bar{\odot}(w, h)\right]\right\} \\
& =\left\{(w, h) \in \mathcal{L}:\left(v_{1}+v_{2}{ }^{g_{1}}, g_{1} g_{2}\right) \bar{\odot}(w, h)=\left(v_{1}, g_{1}\right) \bar{\odot}\left[\left(v_{2}+w^{g_{2}}, g_{2} h\right)\right\}\right. \\
& =\left\{(w, h) \in \mathcal{L}:\left(v_{1}+v_{2}{ }^{g_{1}}+w^{g_{1} g_{2}},\left(g_{1} g_{2}\right) h\right)=\left(v_{1}+v_{2}{ }^{g_{1}}+w^{g_{2} g_{1}}, g_{1}\left(g_{2} h\right)\right)\right\} \\
& =\left\{(w, h) \in \mathcal{L}: w^{g_{1} g_{2}}=w^{g_{2} g_{1}}\right\} .
\end{aligned}
$$

$w^{g_{1} g_{2}}=w^{g_{2} g_{1}}$ for all $g_{1}, g_{2} \in G$, so $w=0$. On the other hand, $w^{g_{1} g_{2}}=w^{g_{2} g_{1}}$ is independent from $h$, so $h$ can be anything in $G$, thus $N_{r}(\mathcal{L})=\{0\} \times G$.

The nucleus of $\mathcal{L}$ is the intersection of left, right and middle nuclei. Therefore, $N(\mathcal{L})=\{0\} \times Z(G)$.

Finally, let $(w, h)$ be in the center of $\mathcal{L}$, then $(w, h)$ is in the nucleus of $\mathcal{L}$. Therefore $w=0$ and $h \in Z(G)$, but $(0, h)$ is also in the centrum, hence $(0, h) \bar{\odot}(v, g)=(v, g) \bar{\odot}(0, h)$ for all $(v, g) \in \mathcal{L}$. That is $\left(v^{h}, h g\right)=(v, g h)$ if and only if $v^{h}=v$ for all $v \in V$ if and only if $h$ is the identity operator in $G$. Therefore, $Z(\mathcal{L})=\left\{\left(0, i d_{V}\right)\right\}$ where $i d_{V}$ is the identity map from $V$ to $V$.

Example 3.5. Let $G L(\mathcal{H})$ be the group of invertible operators inside the space of bounded linear operators $L(\mathcal{H})$, where $\mathcal{H}$ is an infinite dimensional Hilbert space over $\mathbb{C}$. The infinite dimensional Hilbert space $\mathcal{H}$ over $\mathbb{C}$ is an additive group with the neutral element 0 . It is an infinite dimensional manifold since it is locally homeomorphic to itself, and the addition and inversion maps are smooth. On the other hand, the group of invertible operators $G L(\mathcal{H})$ is open in $L(\mathcal{H})$ with respect to operator norm, so it is a Banach-Lie group. Therefore, $\mathcal{H} \times G L(\mathcal{H})$ is a smooth manifold as a cartesian product of smooth manifolds. Furthermore, if the evaluation map ev $: \mathcal{H} \times G L(\mathcal{H}) \rightarrow \mathcal{H} ;(h, T) \mapsto h^{T}$ is smooth, then the twisted semidirect product, the left and the right division maps are smooth, hence $(\mathcal{H} \times G L(\mathcal{H}), \bar{\odot})$ is an infinite dimensional left Bol Lie loop by corollary 3.4.1.

Lemma 3.6. Any Lie algebra $\mathfrak{l}$, is an Akivis algebra with the trilinear operation defined by $\langle x, y, z\rangle: \mathfrak{l} \times \mathfrak{l} \times \mathfrak{l} \rightarrow \mathfrak{l}$; $(x, y, z) \mapsto[[x, y], z]-[x,[y, z]]$.

Proof. Let $\mathfrak{l}$ be a Lie algebra, then there exists a bilinear skew-symmetric operation, [.,.] $: \mathfrak{l} \times \mathfrak{l} \rightarrow \mathfrak{l} ;(x, y) \mapsto[x, y]$. To see that $\mathfrak{l}$ is indeed an Akivis algebra, we need to verify the Akivis identity: $[[x, y], z]+[[y, z], x]+[[z, x], y]=\alpha-\beta$ where $\alpha$ and $\beta$ given below.

$$
\begin{aligned}
& \alpha=\langle x, y, z\rangle+\langle y, z, x\rangle+\langle z, x, y\rangle \\
& \beta=\langle x, z, y\rangle+\langle y, x, z\rangle+\langle z, y, x\rangle
\end{aligned}
$$

Let $\gamma=[[x, y], z]+[[y, z], x]+[[z, x], y]$, then we want to show that $\gamma=\alpha-\beta$. Since $\mathfrak{l}$ is a Lie algebra it satisfies the Jacobi identity and this forces that $\gamma=0$, hence we only need to show that $\alpha=\beta$

$$
\begin{aligned}
\alpha & =[[x, y], z]-[x,[y, z]]+[[y, z], x]-[y,[z, x]]+[[z, x], y]-[z,[x, y]] \\
& =([[x, y], z]+[[y, z], x]+[[z, x], y])-([x,[y, z]]+[y,[z, x]]+[z,[x, y]]) \\
& =2([[x, y], z]+[[y, z], x]+[[z, x], y]) \\
& =2(0)=0
\end{aligned}
$$

We can similarly show that $\beta=0$, so $\alpha=\beta=0$. Therefore, any Lie algebra $l$ is an Akisvis algebra if the trilinear operation defined as in Lemma 3.6. 
Theorem 3.7. Let $\mathfrak{h}$ and $\mathfrak{k}$ be two Lie algebras over the field $\mathbb{F}$, and let $\rho: \mathfrak{h} \rightarrow \operatorname{Der}_{\mathbb{F}}(\mathfrak{k})$ be a Lie algebra homomorphism. Then,

1. $\mathfrak{l}=\mathfrak{k} \bar{\rtimes}_{\rho} \mathfrak{h}$ is an Akivis algebra with bracket and trilinear operations given in (48) and (49) below respectively.

$$
\begin{aligned}
{\left[\left(k_{1}, h_{1}\right),\left(k_{2}, h_{2}\right)\right] } & =\left(\left[k_{1}, k_{2}\right]+\left(k_{2}\right)^{\left(h_{1}\right) \rho}-\left(k_{1}\right)^{\left(h_{2}\right) \rho},\left[h_{1}, h_{2}\right]\right) \text { for all } k_{1}, k_{2} \in \mathfrak{k} \text { and } h_{1}, h_{2} \in \mathfrak{h} . \\
\langle x, y, z\rangle & =[[x, y], z]-[x,[y, z]] \text { for all } x, y, z \in \mathfrak{l} .
\end{aligned}
$$

2. $\overline{\mathfrak{k}}=\{(k, 0): k \in \mathfrak{k}\} \cong \mathfrak{k}$ is an ideal of $\mathfrak{l}$, i.e., $[\overline{\mathfrak{k}}, \mathfrak{l}] \subseteq \overline{\mathfrak{k}}$.

3. $\overline{\mathfrak{h}}=\{(0, h): h \in \mathfrak{h}\} \cong \mathfrak{h}$ is a subalgebra of $\mathfrak{l}$, i.e., $[\overline{\mathfrak{h}}, \overline{\mathfrak{h}}] \subseteq \overline{\mathfrak{h}}$.

Proof. 1. The bracket on $\mathfrak{l}$ is skew symmetric as follows:

$$
\begin{aligned}
& {\left[\left(k_{1}, h_{1}\right),\left(k_{2}, h_{2}\right)\right]=\left(\left[k_{1}, k_{2}\right]+\left(k_{2}\right)^{\left(h_{1}\right) \rho}-\left(k_{1}\right)^{\left(h_{2}\right) \rho},\left[h_{1}, h_{2}\right]\right)} \\
& \left.=\left(-\left[k_{2}, k_{1}\right]-\left(\left(k_{1}\right)^{\left(h_{2}\right) \rho}-\left(k_{2}\right)^{\left(h_{1}\right.}\right) \rho\right),-\left[h_{2}, h_{1}\right]\right) \\
& =-\left(\left[k_{2}, k_{1}\right]+\left(k_{1}\right)^{\left(h_{2}\right) \rho}-\left(k_{2}\right)^{\left(h_{1}\right) \rho},\left[h_{2}, h_{1}\right]\right) \\
& =-\left[\left(k_{2}, h_{2}\right),\left(k_{1}, h_{1}\right)\right]
\end{aligned}
$$

The bracket on $\mathfrak{l}$ is bilinear since the bracket operations on $\mathfrak{k}$ and $\mathfrak{h}$ are bilinear. On the other hand $(h) \rho$ is linear for each $h \in \mathfrak{h}$. Therefore, we only need to verify the Jacobi identity on $\mathfrak{l}$, and this can be seen as follow: Let $x=\left[\left[\left(k_{1}, h_{1}\right),\left(k_{2}, h_{2}\right)\right],\left(k_{3}, h_{3}\right)\right], y=\left[\left[\left(k_{2}, h_{2}\right),\left(k_{3}, h_{3}\right)\right],\left(k_{1}, h_{1}\right)\right]$, and $z=\left[\left[\left(k_{3}, h_{3}\right),\left(k_{1}, h_{1}\right)\right],\left(k_{2}, h_{2}\right)\right]$. We want to show that $x+y+z=(0,0)$. Notice that:

$$
\begin{aligned}
x & =\left[\left[\left(k_{1}, h_{1}\right),\left(k_{2}, h_{2}\right)\right],\left(k_{3}, h_{3}\right)\right] \\
& =\left[\left(\left[k_{1}, k_{2}\right]+\left(k_{2}\right)^{\left(h_{1}\right) \rho}-\left(k_{1}\right)^{\left(h_{2}\right) \rho},\left[h_{1}, h_{2}\right]\right),\left(k_{3}, h_{3}\right)\right] \\
& =\left(\left[\left[k_{1}, k_{2}\right]+\left(k_{2}\right)^{\left(h_{1}\right) \rho}-\left(k_{1}\right)^{\left(h_{2}\right) \rho}, k_{3}\right]+\left(k_{3}\right)^{\left(\left[h_{1}, h_{2}\right] \rho \rho\right.}-\left(\left[k_{1}, k_{2}\right]+\left(k_{2}\right)^{\left(h_{1}\right) \rho}-\left(k_{1}\right)^{\left(h_{2}\right) \rho}\right)^{\left(h_{3}\right) \rho},\left[\left[h_{1}, h_{2}\right], h_{3}\right]\right) \\
& =\left(\left[\left[k_{1}, k_{2}\right], k_{3}\right]+\left[k_{2}{ }^{\left(h_{1}\right) \rho}, k_{3}\right]-\left[k_{1}^{\left(h_{2}\right) \rho}, k_{3}\right]+k_{3}{ }^{\left(\left[h_{1}, h_{2}\right]\right) \rho}-\left[k_{1}, k_{2}\right]^{\left(h_{3}\right) \rho}-k_{2}^{\left(h_{1}\right) \rho\left(h_{3}\right) \rho}+k_{1}^{\left(h_{2}\right) \rho\left(h_{3}\right) \rho},\left[\left[h_{1}, h_{2}\right], h_{3}\right]\right) .
\end{aligned}
$$

The map $\rho$ is a Lie algebra homomorphism, so $\left(\left[h_{1}, h_{2}\right]\right) \rho=\left[\left(h_{1}\right) \rho,\left(h_{2}\right) \rho\right]$, and $\left[\left(h_{1}\right) \rho,\left(h_{2}\right) \rho\right]=\left(h_{2}\right) \rho\left(h_{1}\right) \rho-\left(h_{1}\right) \rho\left(h_{2}\right) \rho$ since $\operatorname{Der}_{\mathbb{F}}(\mathfrak{k})$ is a Lie algebra with $[f, g]=g f-f g$ for each $f, g \in \operatorname{Der}_{\mathbb{F}}(\mathfrak{k})$. Therefore,

$$
k_{3}{ }^{\left(\left[h_{1}, h_{2}\right]\right) \rho}=k_{3}^{\left(h_{2}\right) \rho\left(h_{1}\right) \rho}-k_{3}{ }^{\left(h_{1}\right) \rho\left(h_{2}\right) \rho}
$$

On the other hand, $(h) \rho$ is a derivation on $\mathfrak{k}$, so $(h) \rho$ preserves the Leibniz rule, and this gives that:

$$
\left[k_{1}, k_{2}\right]^{\left(h_{3}\right) \rho}=\left[k_{1}{ }^{\left(h_{3}\right) \rho}, k_{2}\right]+\left[k_{1}, k_{2}{ }^{\left(h_{3}\right) \rho}\right]
$$

If we let $x=\left(x_{1}, x_{2}\right)$, then $x_{1}$ and $x_{2}$ coordinates are written as follow.

$$
\begin{gathered}
x_{1}=\left[\left[k_{1}, k_{2}\right], k_{3}\right]+\left[k_{2}{ }^{\left(h_{1}\right) \rho}, k_{3}\right]-\left[k_{1}{ }^{\left(h_{2}\right) \rho}, k_{3}\right]+k_{3}{ }^{\left(h_{2}\right) \rho\left(h_{1}\right) \rho}-k_{3}{ }^{\left(h_{1}\right) \rho\left(h_{2}\right) \rho}-\left[k_{1}{ }^{\left(h_{3}\right) \rho}, k_{2}\right]-\left[k_{1}, k_{2}{ }^{\left(h_{3}\right) \rho}\right]-k_{2}{ }^{\left(h_{1}\right) \rho\left(h_{3}\right) \rho}+k_{1}{ }^{\left(h_{2}\right) \rho\left(h_{3}\right) \rho} \\
x_{2}=\left[\left[h_{1}, h_{2}\right], h_{3}\right]
\end{gathered}
$$

We can similarly find $y=\left(y_{1}, y_{2}\right)$ and $z=\left(z_{1}, z_{2}\right)$ such that

$$
\begin{gathered}
y_{1}=\left[\left[k_{2}, k_{3}\right], k_{1}\right]+\left[k_{3}{ }^{\left(h_{2}\right) \rho}, k_{1}\right]-\left[k_{2}{ }^{\left(h_{3}\right) \rho}, k_{1}\right]+k_{1}{ }^{\left(h_{3}\right) \rho\left(h_{2}\right) \rho}-k_{1}{ }^{\left(h_{2}\right) \rho\left(h_{3}\right) \rho}-\left[k_{2}{ }^{\left(h_{1}\right) \rho}, k_{3}\right]-\left[k_{2}, k_{3}{ }^{\left(h_{1}\right) \rho}\right]-k_{3}{ }^{\left(h_{2}\right) \rho\left(h_{1}\right) \rho}+k_{2}{ }^{\left(h_{3}\right) \rho\left(h_{1}\right) \rho} \\
\left.y_{2}=\left[\left[h_{2}, h_{3}\right], h_{1}\right]\right) \\
z_{1}=\left[\left[k_{3}, k_{1}\right], k_{2}\right]+\left[k_{1}{ }^{\left(h_{3}\right) \rho}, k_{2}\right]-\left[k_{3}{ }^{\left(h_{1}\right) \rho}, k_{2}\right]+k_{2}{ }^{\left(h_{1}\right) \rho\left(h_{3}\right) \rho}-k_{2}{ }^{\left(h_{3}\right) \rho\left(h_{1}\right) \rho}-\left[k_{3}{ }^{\left(h_{2}\right) \rho}, k_{1}\right]-\left[k_{3}, k_{1}{ }^{\left(h_{2}\right) \rho}\right]-k_{1}{ }^{\left(h_{3}\right) \rho\left(h_{2}\right) \rho}+k_{3}{ }^{\left(h_{1}\right) \rho\left(h_{2}\right) \rho} \\
z_{2}=\left[\left[h_{3}, h_{1}\right], h_{2}\right]
\end{gathered}
$$

The second coordinate of $x+y+z$ is $x_{2}+y_{2}+z_{2}=\left[\left[h_{1}, h_{2}\right], h_{3}\right]+\left[\left[h_{2}, h_{3}\right], h_{1}\right]+\left[\left[h_{3}, h_{1}\right], h_{2}\right]=0$ since $\mathfrak{h}$ is a Lie algebra. Moreover, a part of the first coordinate of $x+y+z$ is $\left[\left[k_{2}, k_{3}\right], k_{1}\right]+\left[\left[k_{2}, k_{3}\right], k_{1}\right]+\left[\left[k_{3}, k_{1}\right], k_{2}\right]=0$ since $\mathfrak{k}$ is a Lie algebra. The rest of the first coordinate of $x+y+z$ is rewritten as: 
$\left(\left[k_{2}{ }^{\left(h_{1}\right) \rho}, k_{3}\right]-\left[k_{2}{ }^{\left(h_{1}\right) \rho}, k_{3}\right]\right)+\left(-\left[k_{1}{ }^{\left(h_{2}\right) \rho}, k_{3}\right]-\left[k_{3}, k_{1}{ }^{\left(h_{2}\right) \rho}\right]\right)+\left(k_{3}{ }^{\left(h_{2}\right) \rho\left(h_{1}\right) \rho}-k_{3}{ }^{\left(h_{2}\right) \rho\left(h_{1}\right) \rho}\right)+\left(-k_{3}{ }^{\left(h_{1}\right) \rho\left(h_{2}\right) \rho}+k_{3}{ }^{\left(h_{1}\right) \rho\left(h_{2}\right) \rho}\right)$ $+\left(-\left[k_{1}{ }^{\left(h_{3}\right) \rho}, k_{2}\right]+\left[k_{1}{ }^{\left(h_{3}\right) \rho}, k_{2}\right]\right)+\left(-\left[k_{1}, k_{2}{ }^{\left(h_{3}\right) \rho}\right]-\left[k_{2}{ }^{\left(h_{3}\right) \rho}, k_{1}\right]\right)+\left(-k_{2}{ }^{\left(h_{1}\right) \rho\left(h_{3}\right) \rho}+k_{2}{ }^{\left(h_{1}\right) \rho\left(h_{3}\right) \rho}\right)+\left(k_{1}{ }^{\left(h_{2}\right) \rho\left(h_{3}\right) \rho}-k_{1}{ }^{\left(h_{2}\right) \rho\left(h_{3}\right) \rho}\right)$ $+\left(\left[k_{3}{ }^{\left(h_{2}\right) \rho}, k_{1}\right]-\left[k_{3}{ }^{\left(h_{2}\right) \rho}, k_{1}\right]\right)+\left(k_{1}{ }^{\left(h_{3}\right) \rho\left(h_{2}\right) \rho}-k_{1}{ }^{\left(h_{3}\right) \rho\left(h_{2}\right) \rho}\right)+\left(k_{2}{ }^{\left(h_{3}\right) \rho\left(h_{1}\right) \rho}-k_{2}{ }^{\left(h_{3}\right) \rho\left(h_{1}\right) \rho}\right)$, and this sum is zero since the sum in each parentheses is zero. Therefore, $x+y+z=(0,0)$, and we conclude that the twisted semidirect product of Lie algebras is a Lie algebra. Therefore, $\mathfrak{l}$ is an Akivis Algebra by Lemma 3.6.

2. The first claim, $\mathfrak{k} \cong \overline{\mathfrak{k}}$, is clear. We will show that $[\overline{\mathfrak{k}}, \mathfrak{l}] \subseteq \overline{\mathfrak{k}}$. Let $(k, 0) \in \overline{\mathfrak{k}}$, and let $\left(k^{*}, h\right) \in \mathfrak{l}$. Then, $\left[(k, 0),\left(k^{*}, h\right)\right]=$ $\left(\left[k, k^{*}\right]+\left(k^{*}\right)^{(0) \rho}-(k)^{(h) \rho},[0, h]\right)$ where $\left(k^{*}\right)^{(0) \rho}=\left(k^{*}\right)^{i d}=k^{*}$ and $[0, h]=0$, so $\left[(k, 0),\left(k^{*}, h\right)\right]=\left(\left[k, k^{*}\right]-k^{*}+\left((k)^{(h) \rho}, 0\right)=\right.$ $\left(k^{* *}, 0\right) \in \overline{\mathfrak{k}}$, where $k^{* *}=\left[k, k^{*}\right]-k^{*}+(k)^{(h) \rho}$. Therefore, $\mathfrak{k}$ is an ideal of $\mathfrak{l}$.

3. $\mathfrak{h} \cong \overline{\mathfrak{h}}$ is clear. Let $(0, h),\left(0, h^{*}\right) \in \overline{\mathfrak{h}}$, then $\left[(0, h),\left(0, h^{*}\right)\right]=\left([0,0]+(0)^{(h) \rho}-(0)^{\left(h^{*}\right) \rho},\left[h, h^{*}\right]\right)=\left(0, h^{* *}\right) \in \overline{\mathfrak{h}}$. Therefore, $[\overline{\mathfrak{h}}, \overline{\mathfrak{h}}] \subseteq \overline{\mathfrak{h}}$, so $\mathfrak{h}$ is a subalgebra of $\mathfrak{l}$.

\section{Acknowledgements}

This paper comes from a part of the author's doctoral dissertation from Western Michigan University, Kalamazoo, Michigan. Therefore, author would like to thank to his advisor Clifton Ealy for his contributions and supports. The author also thanks the editor and the anonymous reviewers for their helpful and constructive comments that greatly contributed to improving the final version of this paper.

\section{References}

Figula, A., \& Strambach, K. (2007). Loops which are semidirect products of groups. Acta Mathematica Hungarica, 114(3), 247-266. http://dx.doi.org/10.1007/s10474-006-0529-3

Greer, M., \& Raney, L. (2014). Moufang semidirect products of loops with groups and inverse property extensions. Comment.Math.Univ.Carolin., 55(3), 411-420.

Hall, M. (1999). The theory of groups. Providence, R.I, AMS Chelsea Pub.

Hein, W. (1990). Einfuhrung in die Struktur- und Darstellungstheorie der Klassischen Gruppen. Berlin, Springer-Verlag.

Humphreys, J. E. (1972). Introduction to Lie algebras and representation theory. New York, Springer-Verlag.

Johnson, K. W., \& Sharma, B. (1980). On a family of Bol loops. Boll. Un. Math. Ital. (Alg. e Geo. Suppl.), 2,119-126.

Johnson, K. W.,\& Smith, J. D. (2010). Matched Pairs, Permutation Representations, and the Bol Property. Communications in Algebra, 38(8), 2903-2914. https://doi.org/10.1080/00927870903095590

Kiechle, H. (2002). Theory of K-loops. Berlin, Springer.

Kinyon, M. K., \& Jones, O. (2000). Loops and semidirect products. Communications in Algebra, $28(9), 4137-4164$. https://doi.org/10.1080/00927870008827079

Knapp, A. (2016). Representation Theory of Semisimple Groups. Princeton, N. J, Princeton University Press.

Nagy, P. T., \& Strambach, K. (2002). Loops in group theory and Lie theory. Berlin, New York, Walter de Gruyter.

Nagy, P. T., \& Strambach, K. (2008). Schreier loops. Czechoslovak Mathematical Journal, 58(3), $759-786$. https://doi.org/10.1007/s10587-008-0050-7

Pflugfelder, H. (1990). Quasigroups and loops: Introduction. Heldermann, Berlin.

Robinson, D. A. (1966). Bol loops. Transactions of the American Mathematical Society, 123(2), 341-341. https://doi.org/10.1090/s0002-9947-1966-0194545-4

Sepanski, M. R. (2007). Compact Lie groups. New York, NY: Springer.

Wallach, N. R. (1988). Real Reductive Groups I. Boston: Academic Press.

\section{Copyrights}

Copyright for this article is retained by the author(s), with first publication rights granted to the journal.

This is an open-access article distributed under the terms and conditions of the Creative Commons Attribution license (http://creativecommons.org/licenses/by/4.0/). 